\title{
Acoustic Output of Multi-Line Transmit Beam Forming for Fast Cardiac Imaging: A Simulation Study
}

\author{
Pedro Santos, Student Member, IEEE, Ling Tong, Student Member, IEEE, \\ Alejandra Ortega, Student Member, IEEE, Lasse Løvstakken, Member, IEEE, Eigil Samset \\ and Jan D'hooge, Member, IEEE
}

\begin{abstract}
Achieving higher frame rates in cardiac ultrasound could unveil short-lived myocardial events and lead to new insights on cardiac function. Multi-Line Transmit (MLT) beam forming (i.e. simultaneously transmitting multiple focused beams) is a potential approach to achieve this. However, two challenges come with it: firstly, it leads to cross-talk between the MLT beams, appearing as imaging artifacts; and secondly, it presents acoustic summation in the near field, where multiple MLT beams overlap. Although several studies have focused on the former, no studies have looked into the implications of the latter on acoustic safety. In this paper, the acoustic field of 4-MLT was simulated and compared with Single-Line Transmit (SLT). The findings suggest that standard MLT does present potential concerns. Compared to SLT, it shows a two-fold increase in Mechanical Index (MI) (from 1.0 to 2.3), a six-fold increase in spatial-peak pulseaverage intensity $\left(I_{\text {sppa }}\right.$ ) (from 99 to $576 \mathrm{~W} \cdot \mathrm{cm}^{-2}$ ) and a twelvefold increase in spatial-peak temporal-average intensity $\left(I_{\text {spta }}\right)$ (from 119 to $1407 \mathrm{~mW} \cdot \mathrm{cm}^{-2}$ ). Subsequently, modifications of the transmit pulse and delay line of MLT were studied. These modifications allowed for a change in the spatio-temporal distribution of the acoustic output, thereby significantly decreasing the safety indices $\left(M I=1.2, I_{\text {sppa }}=92 \mathrm{~W} \cdot \mathrm{cm}^{-2}\right.$ and $\left.I_{\text {spta }}=366 \mathrm{~mW} \cdot \mathrm{cm}^{-2}\right)$. Accordingly, they help mitigate the concerns around MLT, reducing potential trade-offs between acoustic safety and image quality.
\end{abstract}

Index Terms-Multi-Line Transmit, beam forming, acoustic safety, mechanical index, thermal index, acoustic intensity.

\section{INTRODUCTION}

$\mathbf{U}$ LTRASOUND imaging has become the preferred modality to assess the anatomy and function of the human heart in clinical practice. Nonetheless, commercially available echocardiographic imaging systems operate on a basis of $c a$. 100 frames per second (FPS) for 2D imaging and considerably less for 3D. This is insufficient to assess complex and shortlived myocardial events [1] and a wide variety of cardiac studies would benefit from improved temporal resolution [2], [3], e.g. elasticity imaging [4], myocardial function estimation [5], complex blood flow imaging [6] and mechanical activation imaging [7]. Several fast acquisition techniques have thus been proposed over the past three decades.

P. Santos, L. Tong, A. Ortega and J. D'hooge are with Department of Cardiovascular Sciences, KU Leuven, Leuven, Belgium, (e-mail: pedro.santos@med.kuleuven.be).

L. Løvstakken and J. D'hooge are with the Medical Imaging Lab (MI-Lab), Norwegian University of Science and Technology, Trondheim, Norway.

P. Santos, L. Løvstakken and E. Samset are with the GE Vingmed Ultrasound AS, Horten, Norway

E. Samset is also with the University of Oslo, Oslo, Norway and the Center for Cardiological Innovation, Oslo, Norway.
Initially, multiple adjacent image lines were reconstructed from each transmit beam - Multi-Line Acquisition (MLA) [8]. Moreover, narrow-sector imaging [9], sparse sector scanning [1] and ECG-gating [10] have been proposed. MLA has also been extended to large insonifications, i.e. plane waves [11] or diverging waves [12], benefiting from coherent-compounding [13], [14]. Yet another approach uses multiple focused beams in the same transmit event - Multi-Line Transmit (MLT) [15]. An overview on the principles and applications of fast cardiac imaging can be found in [2].

MLT beam forming reduces the number of transmit events while preserving lateral resolution and the capability of performing harmonic imaging [16], contrarily to broad transmit beams. Each MLT beam can be transmitted from either interleaved transducer elements [15] or semi-apertures [17] or by linearly summing a plurality of delay lines [18]. Nevertheless, parallel transmit inherently presents beam-to-beam interference, seen in the image as cross-talk artifacts. This was a reason for parallel receive beam forming to have been preferred over MLT. However, it has recently been shown that MLT can achieve image quality comparable to the one of conventional Single-Line transmit (SLT) [19]-[22]. Nonetheless, few studies have looked into the other side of acoustic interference, related to acoustic safety. As multiple beams overlap in the near field, MLT leads to acoustic summation, potentially exceeding the limits for its use in medical imaging.

According to the Output Display Standard, safety of medical ultrasound imaging is indicated by the mechanical index (MI) and the thermal index (TI) [23], which within recommended limits prevent hazards such as cavitation or teratogenic effects [24]. These are required to be computed and shown on the system for every ultrasonic sequence [25]. Additionally, the US Food and Drug Administration (FDA) requires the calculation of the spatial-peak pulse-average intensity $\left(\mathrm{I}_{\text {sppa }}\right)$ and the spatial-peak temporal-average intensity $\left(\mathrm{I}_{\text {spta }}\right)$, related to mechanical and thermal effects, respectively [26].

The primary aim of this study was therefore to quantify the acoustic output of MLT beam forming according to the parameters presented above. A clinical implementation of ultrasound imaging must obey the safety standards, and thus acoustic summation may require a reduction in transmit power, ultimately meaning a decrease in signal-to-noise ratio (SNR). Beam forming modifications have been proposed to address different challenges, e.g. to suppress cross-talk artifacts [19], 


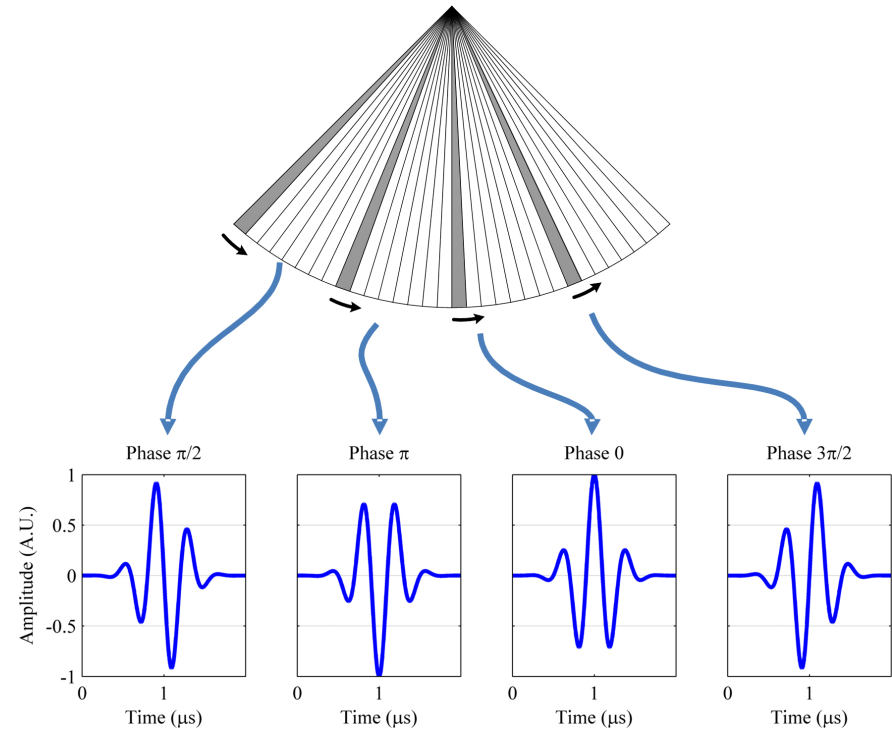

Fig. 1. Phased 4-MLT: a different phase is assigned to the pulse of each MLT beam and each of these beams will cover a sub-sector of the imaging sector. In the configuration shown, the phases used for each of the 4 MLT beams were: $\frac{\pi}{2}, \pi, 0$ and $\frac{3 \pi}{2}$.

[27]. Alike, we studied modifications of the standard MLT beam forming approach that could mitigate these issues [28].

\section{METHODS}

In the present study conventional SLT, standard 4-MLT and two modifications of 4-MLT beam forming were simulated. Additionally, both modifications were combined to assess to which extent they could further decrease acoustic output. The modifications are described later in this section, as well as the acoustic parameters used to evaluate the resulting acoustic fields.

\section{A. Simulation Setup}

The 1-D cardiac phased array used is described in Table I whereas the system settings are presented in Table II. In order to study the influence of the scanning geometry on the proposed modifications, two different angles between MLT beams were implemented. Simulations were performed using an in-house developed linear simulator based on the spatial

TABLE I

UltRASONIC TRANSDUCER CONFIGURATION

\begin{tabular}{ll}
\hline Aperture size & $18.56 \mathrm{~mm}$ \\
Number of crystals & 64 \\
Crystal height & $13 \mathrm{~mm}$ \\
Pitch & $290 \mu \mathrm{m}$ \\
Kerf & $0 \mathrm{~mm}$ \\
Tx pulse central frequency & $2.5 \mathrm{MHz}$ \\
Tx pulse bandwidth & $63 \%(-6 \mathrm{~dB})$ \\
Tx pulse length & $2 \mu \mathrm{s}$ \\
\hline Tx = Transmit. &
\end{tabular}

impulse response method - Simpulse - using a sampling rate of $50 \mathrm{MHz}$ and an anti-aliasing frequency for the excitation pulse of $8.3 \mathrm{MHz}$ [29]. Accuracy in the near field is of great relevance for the present study, as MLT beams mostly overlap close to the transducer. Such accuracy is assured even at low sampling rates by the use of the analytically smoothed impulse response.

To account for ultrasonic attenuation in the tissue, acoustic pressures were derated assuming an acoustic attenuation coefficient of $0.3 \mathrm{~dB} \mathrm{~cm}^{-1} \mathrm{MHz}^{-1}$, as used when measuring acoustic output parameters [25]. The transmit voltage for SLT simulations was defined as the one leading to an MI of 1.0. This same transmit voltage was used for each of the multiple pulses in MLT simulations.

To compare SLT and standard MLT beam forming, an observation grid of $\pm 35 \mathrm{~mm}$-azimuth by $100 \mathrm{~mm}$-depth was used. However, as most of the MLT beam-to-beam interference occurs in the near field, the study of the MLT modifications was limited to a region of $\pm 13 \mathrm{~mm}$-azimuth by $18 \mathrm{~mm}$-depth.

\section{B. MLT Configurations}

1) Standard MLT: 4-MLT was implemented by dividing the imaging sector into four equally sized sub-sectors (Fig. 1). Four delay lines that would conventionally be used in independent SLT transmit events were linearly summed to create an MLT transmit event. The angle between MLT beams was defined as the angle of the sub-sectors and each MLT beam images one of the sub-sectors. Moreover, Tukey $(\alpha=0.5)$ apodization was used on transmit for all MLT simulations. This has been shown to be required for crosstalk suppression in MLT beam forming [30]. On the contrary, rectangular apodization was used for SLT as no cross-talk is present.

2) Phased MLT: In the standard implementation, the transmit pulse waveform is the same for all MLT beams. Accordingly, constructive interference can occur at positions where the MLT beams overlap, thereby leading to a significant increase in acoustic pressure. However, a modification where a different pulse phase is given to each MLT beam, would allow for an immediate change of the interference pattern. This change could in theory decrease the acoustic pressure of the combined ultrasonic beam.

TABLE II

SYSTEM CONFIGURATION

\begin{tabular}{|c|c|c|c|}
\hline & SLT & 4-MLT wide $_{\text {}}$ & 4-MLT ${ }_{\text {narrow }}$ \\
\hline Angle between MLT beams $\left({ }^{\circ}\right)$ & - & 22.73 & 15.19 \\
\hline Imaging sector $\left(^{\circ}\right)$ & 90.46 & 90.46 & 60.30 \\
\hline Number of Tx lines & 96 & 96 & 64 \\
\hline Number of Tx events & 96 & 24 & 16 \\
\hline Focal depth $(\mathrm{cm})$ & 6 & 6 & 6 \\
\hline Imaging depth $(\mathrm{cm})$ & 15 & 15 & 15 \\
\hline Frame rate (FPS) & 53 & 214 & 321 \\
\hline
\end{tabular}

SLT $=$ Single-Line Transmit; MLT $=$ Multi-Line Transmit.

$\mathrm{Tx}=$ Transmit Rx $=$ Receive . 


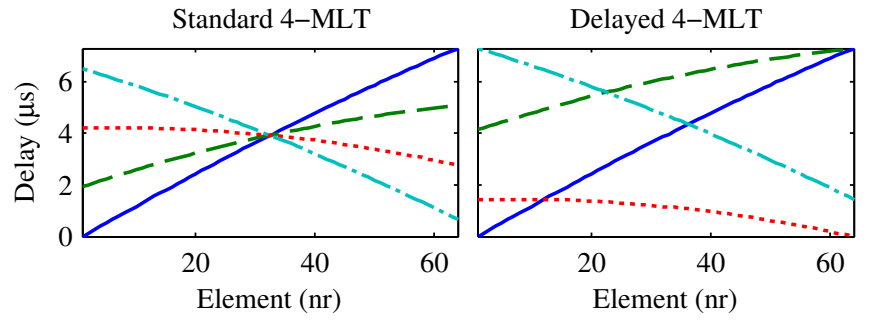

Fig. 2. 4-MLT delay lines: in the standard configuration (left), all lines intersect at the centre of the array, whereas in the modified one (right), a small delay may be added/subtracted to each delay line of a given transmit event. Here, the outermost oriented delay line (continuous line) was kept constant, while 2 MLT delay lines (dashed lines) were aligned at the end of the transmit event and the remaining one (dotted line) was aligned at its beginning.

To study this modification, a Gaussian pulse was defined:

$$
v(t)=a e^{-\frac{\left(t-t_{0}\right)^{2}}{2 \sigma^{2}}} \cos \left(2 \pi f_{0} t+\varphi_{0}\right)
$$

and the phase $\varphi_{0}$ was defined as shown in Table III, with 11 configurations having a different phase for each MLT beam and 3 having the same phase, as means of comparison. A schematic representation is given in Fig. 1.

3) Delayed MLT: In standard MLT, all the delay lines of a given transmit event have the same time delay value for the central element of the transducer (Fig. 2 - left panel). This holds true if the focal depth (i.e. the distance from the centre of the transducer to the focal point) is kept constant for all the MLT beams. Therefore, in standard MLT all the simultaneously-transmitted beams overlap at the central element, increasing the acoustic pressure in tissue in the vicinity of that element.

As a second modification of MLT scanning, the delay lines were reorganised within the transmit event. In practice, a small time delay was added/subtracted to each delay line in order to partially separate the MLT beams in time. The longest delay line (i.e. the delay line of the outermost oriented beam) was kept the same as in the standard MLT configuration, whereas the remaining lines were moved within the limits of the former. As the time interval that the transducer remains in transmit mode (thus not able to receive the echoes reflected from the

TABLE III

Pulse Phase Configurations

\begin{tabular}{ccccc|ccccc}
\hline \multirow{2}{*}{ Config. } & \multicolumn{3}{c|}{ Phase per MLT beam } & \multirow{3}{*}{ Config. } & \multicolumn{4}{c}{ Phase per MLT beam } \\
& $\varphi_{1}$ & $\varphi_{2}$ & $\varphi_{3}$ & $\varphi_{4}$ & & $\varphi_{1}$ & $\varphi_{2}$ & $\varphi_{3}$ & $\varphi_{4}$ \\
\hline 4MLT & 0 & 0 & 0 & 0 & P7 & 0 & $\frac{\pi}{4}$ & $\frac{\pi}{2}$ & $\frac{3 \pi}{4}$ \\
P1 & 0 & $\frac{\pi}{2}$ & $\pi$ & $\frac{3 \pi}{2}$ & P8 & 0 & $\frac{2 \pi}{3}$ & $\frac{4 \pi}{3}$ & 0 \\
P2 & $\frac{3 \pi}{2}$ & $\pi$ & $\frac{\pi}{2}$ & 0 & P9 & $\frac{\pi}{2}$ & $\pi$ & 0 & $\frac{\pi}{2}$ \\
P3 & 0 & $\pi$ & 0 & $\pi$ & P10 & $\frac{\pi}{2}$ & $\pi$ & 0 & $\frac{3 \pi}{2}$ \\
P4 & $\frac{\pi}{2}$ & $\frac{3 \pi}{2}$ & $\frac{\pi}{2}$ & $\frac{3 \pi}{2}$ & P11 & $\pi$ & 0 & 0 & $\pi$ \\
P5 & 0 & $\pi$ & $\frac{\pi}{2}$ & $\frac{3 \pi}{2}$ & P12 & $\pi$ & $\pi$ & $\pi$ & $\pi$ \\
P6 & 0 & $\pi$ & $\pi$ & 0 & P13 & $\frac{\pi}{2}$ & $\frac{\pi}{2}$ & $\frac{\pi}{2}$ & $\frac{\pi}{2}$ \\
\hline
\end{tabular}

$\mathrm{P}_{\mathrm{i}}=$ pulse phase configuration $i$

$\varphi_{\mathrm{i}}=$ Pulse phase of MLT beam $i$ closest scatterers) is defined by the longest delay line, this will not be affected by this modification.

The 13 configurations tested are described in Table IV and an example is schematised in Fig. 2 - right panel.

\section{Acoustic Output Parameters}

The following parameters were used to evaluate the acoustic fields of the tested beam forming methodologies. They were computed from the local instantaneous acoustic pressure $p(\mathbf{r}, t)$ as follows:

1) Mechanical Index: The MI is computed from the attenuated peak-rarefactional acoustic pressure $\left(p_{r}\right)$ at the position $\left(z_{M I}\right)$ of the maximum attenuated pulse-pressure-squared integral (ppsi), according to:

$$
M I=\frac{p_{r}\left(z_{M I}\right) \cdot f_{a w f}{ }^{-1 / 2}}{C_{M I}}
$$

where $f_{a w f}$ is the acoustic-working frequency (measured at the position of the spatial-peak temporal-peak acoustic pressure) and $C_{M I}$ is a normalizing coefficient of $1 \mathrm{MPa} \cdot \mathrm{MHz}^{-1 / 2}$. As $p_{r}$ occurs very close to the transducer surface for MLT, an $f_{a w f}$ of $2.5 \mathrm{MHz}$ was used for all cases.

2) Thermal Index: As the current study targets cardiac applications, the soft tissue TI (TIS) was used. For this model, the power parameter used is the bounded-square output power $\left(P_{1 \times 1}\right)$. This is the maximum value of the time average acoustic output power emitted from any one-centimetre square active area. Limitation of the active area was accomplished by reducing the number of active elements to the central $1 \mathrm{~cm}$-region of the transducer and by resizing the height of the crystals [31]. $P_{1 \times 1}$ was then computed by evaluating the surface integral of the temporal averaged derived instantaneous intensity [32] over an hemisphere of $50 \mathrm{~mm}$ radius located in front of the acoustic transducer:

TABLE IV

DELAY Line CONFIGURATIONS

\begin{tabular}{cl}
\hline Config. & Delay line alignment per MLT beam \\
\hline D1 & all beams aligned at the end of Tx \\
D2 & all beams aligned at the beginning of Tx \\
D3 & $b_{2}$ at the end of Tx and $b_{3}$ at the beginning of Tx \\
D4 & $b_{2}$ aligned at the end of $b_{4}, b_{3}$ aligned at the end of $b_{1}$ \\
D5 & $b_{1}$ and $b_{4}$ at the end of Tx, $b_{2}$ aligned at the beginning of $b_{4}$, \\
$b_{3}$ aligned at the beginning of $b_{1}$
\end{tabular}

$\mathrm{D}_{\mathrm{i}}=$ delay configuration $i, \mathrm{Tx}=$ transmit event, $\mathrm{b}_{\mathrm{n}}=$ MLT beam $n$

Beams not listed follow standard MLT alignment (i.e. central element) 


$$
P_{1 \times 1}=\int_{S} \frac{1}{s r p} \int_{0}^{s r p} \frac{p^{2}(\mathbf{r}, t)}{\rho c} d t d S
$$

where $\operatorname{srp}$ is the scan repetition period, $\rho$ is the tissue density $\left(\rho=1.0 \mathrm{~kg} \cdot \mathrm{m}^{-3}\right)$ and $c$ is the speed of sound $(c=1540 \mathrm{~m}$. $\left.s^{-1}\right)$.

For scanned modes, both at surface and below surface TI values are computed using the same formula:

$$
T I_{a s, s c}=T I_{b s, s c}=\frac{P_{1 \times 1} \cdot f_{a w f}}{C_{T I S, 1}}
$$

where $C_{T I S, 1}$ is a normalizing coefficient of $210 \mathrm{~mW} \cdot \mathrm{MHz}$.

3) Spatial-Peak Pulse-Average Intensity: The $\mathrm{I}_{\text {sppa }}$ is the maximum value of the pulse-average intensity $\left(\mathrm{I}_{\mathrm{pa}}\right)$ in an acoustic field:

$$
I_{\text {sppa }}=\max _{\mathbf{r}}\left\{\frac{1}{t_{d}} \int_{0}^{t_{d}} \frac{p^{2}(\mathbf{r}, t)}{\rho c} d t\right\}
$$

where $I_{p a}$ is the ratio of the pulse intensity integral to the pulse duration $\left(t_{d}\right)$ at a particular point in the acoustic field. In the present study, the pulse intensity integral was approximated as the pulse-pressure-squared integral (ppsi). Moreover, $t_{d}$ is defined as 1.25 times the interval when the ppsi reaches $10 \%$ and $90 \%$ of its final value.

4) Spatial-Peak Temporal-Average Intensity: The $\mathrm{I}_{\text {spta }}$ is the maximum value of the temporal-average intensity $\left(\mathrm{I}_{\mathrm{ta}}\right)$ in an acoustic field:

$$
I_{s p t a}=\max _{\mathbf{r}}\left\{\frac{1}{s r p} \int_{0}^{s r p} \frac{p^{2}(\mathbf{r}, t)}{\rho c} d t\right\}
$$

where $I_{t a}$ is the time average of the instantaneous intensity at a particular point in the acoustic field. Again, instantaneous intensity was approximated by the derived instantaneous intensity (i.e. pulse-pressure-squared).

5) Peak-Compressional Acoustic Pressure: The $\mathrm{p}_{\mathrm{c}}$ is the maximum positive instantaneous acoustic pressure during an acoustic repetition period:

$$
p_{c}=\max _{\mathbf{r}, t}\{p(\mathbf{r}, t)\}
$$

TABLE V

COMBINED CONFIGURATIONS

\begin{tabular}{ccc|ccc}
\hline Config. & Pulse phase & Delay & Config. & Pulse phase & Delay \\
\hline C1 & P1 & D11 & C8 & P8 & D11 \\
C2 & P2 & D11 & C9 & P9 & D11 \\
C3 & P3 & D11 & C10 & P10 & D11 \\
C4 & P4 & D11 & C11 & P11 & D11 \\
C5 & P5 & D11 & C12 & P12 & D11 \\
C6 & P6 & D11 & C13 & P13 & D11 \\
C7 & P7 & D11 & & & \\
\hline
\end{tabular}

$\mathrm{C}_{\mathrm{i}}=$ combined configuration $i$

$\mathrm{P}_{\mathrm{i}}=$ pulse phase configuration $i$ (described in Table III)

$\mathrm{D}_{11}=$ delay configuration 11 (described in Table IV)

\section{RESUltS}

A comparison between the acoustic pressure fields of SLT and standard 4-MLT is given in Fig. 3. Beam-to-beam interference is clearly seen in the region where the MLT beams overlap. Therefore, a significant increase in acoustic pressure was found immediately in front of the ultrasound transducer. Consequently, for MLT beam forming, this becomes the region where either peak-compressional and peak-rarefactional acoustic pressure occur, whereas in SLT they are found around the focal zone. In fact, acoustic pressure more than doubled in the near field and $\mathrm{I}_{\text {sppa }}$ suffered a six-fold increase, whereas $\mathrm{I}_{\text {spta }}$ increased by more than one order of magnitude. On the contrary, due to apodization MLT beam forming resulted in a decrease of $c a .20 \%$ in the pressure around the focal region: from 1.84 MPa in the case of SLT to $1.43 \mathrm{MPa}$ in standard 4MLT. Moreover, both TI and peak-to-peak drive voltage $\left(\mathrm{V}_{\mathrm{pp}}\right)$ were quadrupled. Results are presented in Fig. 4.

Fig. 5 shows the sound field of a given transmit event for all the different pulse phase configurations for the $90^{\circ}$-sector scan. An animated sequence of the complete sector scan can be found here Media-Movie 1. Please note that only the near field is shown ( $\pm 13 \mathrm{~mm}$ in azimuth and $18 \mathrm{~mm}$ in depth). Therefore, the brightest lines are not the actual MLT beams, but the strong acoustic interference between them. As for the acoustic parameters, TI remained fairly the same as in standard 4-MLT with either sector geometry. Conversely, a significant decrease was found for the remaining acoustic parameters. Results are presented in Fig. 4. As expected, the decrease was dependent on the given configuration. The one with the most substantial global decrease (both acoustic pressure and intensity) was the one with pulse phases of $\left[\frac{\pi}{2}-\pi-0-\frac{3 \pi}{2}\right]$ (configuration P10). This was true for both sector opening angles.

Fig. 6 depicts the acoustic pressure field of a transmit event with different delay line configurations. The complete sector scan sequence can be found here Media-Movie 2. Again, note that only the near field is plotted $( \pm 13 \mathrm{~mm}$-azimuth $\times 18 \mathrm{~mm}$ depth region) and thus the brightest lines are the interference between the MLT beams. Quantitatively, delayed-MLT showed a more substantial decrease in the acoustic output parameters than phased MLT. Again, results showed some dependence on the exact delay configuration, whereas the sector geometry had only a small influence (Fig. 4). Nonetheless, no specific configuration was found to lead to the highest decrease in all parameters. As in the previous modification, no significant decrease was found for the TI.

Finally, both modifications were combined. From the previous results, it was clear that shifting the delays influenced the acoustic output in greater extent. As such, one of the best configurations of the delay modification (namely, D11) was combined with all the 13 configurations of phased MLT. Combined configurations are described in Table V. Results are presented in Fig. 4 and showed similar output for all tested configurations, especially in terms of $\mathrm{I}_{\text {spta }}$. Overall, the original D11 was the configuration with lowest MI, while $I_{\text {sppa }}$ and $I_{\text {spta }}$ were slightly reduced in configuration $\mathrm{C} 10 . \mathrm{C} 13$ required the lowest $\mathrm{V}_{\mathrm{pp}}$. 


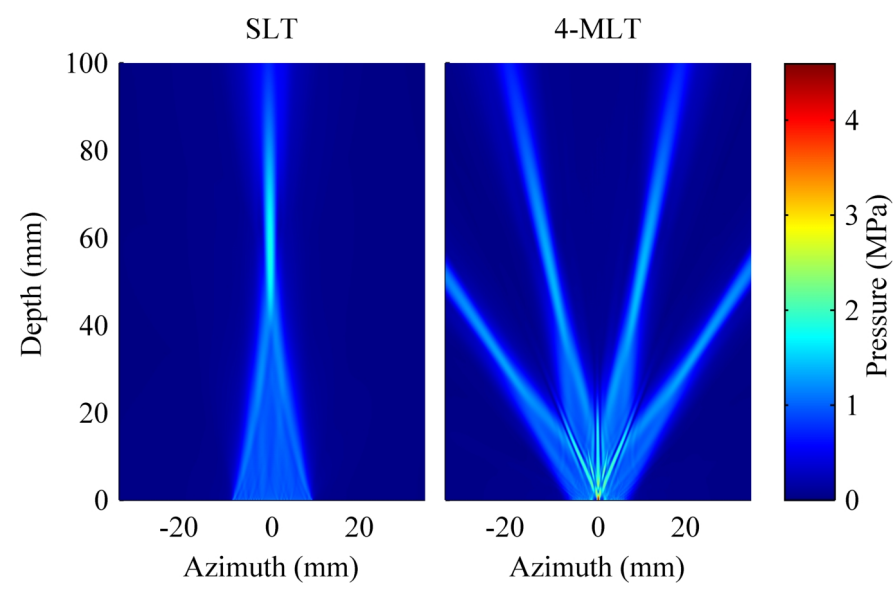

Fig. 3. Comparison of the acoustic pressure fields of SLT and 4-MLT. Strong interference between the multiple beams is found in 4-MLT and the peak acoustic pressure occurs close to the transducer, contrarily to SLT where it occurs around the focal point. Note the difference in acoustic pressure around the focal point, as a consequence of transmit apodization on MLT.

As a comparison, Fig. 7 shows the near field of the acoustic pressure field for a given transmit event and the time averaged intensity for the $90^{\circ}$-sector scan. Four different configurations are depicted: standard 4-MLT, the best phased 4-MLT, the best delayed 4-MLT and the best combined modification. The complete scan sequence can be found here Media-Movie 3.

\section{Discussion}

\section{A. Simulation methods}

In the present study, a linear simulator - Simpulse - was used, thereby intrinsically neglecting non-linear effects (i.e. higher harmonics). Due to the increased attenuation of higher frequencies, such harmonics would lead to an increased loss of energy and thus exacerbate tissue heating. However, nonlinear effects are building up with increasing depth and will thus mainly show further away from the transducer. In this region, MLT and SLT have similar acoustic fields (Fig. 3). As such, the non-linear distortions are likely similar for both modalities, although higher pressures in the near field due to MLT beam overlap could have a small effect.

Another remark is related to the measurement of the acoustic parameters in itself. Traditionally, these are done in the far field, only. In fact, [25] and [26] define a break-point depth of 1.5 times the equivalent transducer diameter and no measurements are done closer than that point. For the transducer simulated, it would be $26 \mathrm{~mm}$, meaning that the potentially hazardous acoustic pressures and intensities that occur in the near field of MLT would be disregarded. However, the results of this study suggest that some care should be taken in the near field when it comes to new beam forming methodologies, as MLT beam forming.

Moreover, it should be noted that intensity computations were done assuming the ultrasonic beams as (locally) plane waves. This is the assumption made for the calculation of the derived instantaneous intensity [32] from either the particle velocity or acoustic pressure, eliminating the requirement of measuring both simultaneously. However, it is valid in the far field only, where pressure and particle velocity are in phase. Close to the transducer, where the MLT acoustic fields are actually more intense, the approximation does not hold and the estimated acoustic intensities are no longer totally accurate. A complementary study on the computation of exact acoustic intensities in the near field of MLT should therefore be conducted. Although it has been reported that the planewave approximation overestimates intensities [33], the extent of this approximation on MLT beam forming is not known.

\section{B. Acoustic output of standard MLT}

Acoustic fields of 4-MLT (Fig. 3) clearly show a strong beam-to-beam interference at the positions where the MLT beams sum coherently. This may be a major concern per se, leading to increased acoustic pressure and intensity. Notwithstanding, the shifting of the peak-compressional acoustic pressure from the focal region to the centre of the transducer brings additional concern when going from SLT to MLT beam forming. In the former, the peak pressure occurs around the focal region, i.e. in the far field. Therefore, it is shifted during the scan sequence, spatially smoothing the temporal-average intensity $\left(\mathrm{I}_{\mathrm{ta}}\right)$. However, in the latter it occurs in front of the ultrasonic transducer for every transmission, regardless of the steering angle. Hence, averaged intensity is substantially increased in this beam forming technique.

As expected, the TI was 4 times higher in 4-MLT than in conventional SLT. As four beams are emitted per transmit event in 4-MLT, the total acoustic output power was expected to be four-folded and so would the TI. Please note that only the central $1 \times 1 \mathrm{~cm}^{2}$ is used for the computation of TI, following the current standards [31]. There, all the elements have unitary apodization, both for SLT and MLT. Moreover, the decrease in acoustic pressure found around the focal region of MLT is in good agreement with the transmit apodization used. In fact, the Tukey $(\alpha=0.5)$ window used in this study had a relative power of 0.74 , whereas the acoustic pressure in the focal region of 4-MLT was 0.77 of the one obtained with SLT beam forming.

In order to assure the safety of the ultrasonic sequences, the MI of an ultrasonic sequence should not exceed the value 1.9, while TI should remain below 6.0 [23]. However, the British Medical Ultrasound Society has published additional guidelines for the TI based on the exposure time [34]. For instance, for cardiac applications, the TI should be below 2.0 if exposure time is 20 minutes, whereas for exposures of less than 1 minute a TI of 4.0 can be reached. As for the $\mathrm{I}_{\text {sppa }}$, it should be lower than $190 \mathrm{~W} \cdot \mathrm{cm}^{-2}$ and $\mathrm{I}_{\text {spta }}$ should be no higher than $720 \mathrm{~mW} \cdot \mathrm{cm}^{-2}$ [26]. ${ }^{1}$

As a consequence of the specificities stated before, the acoustic field of standard 4-MLT exceeds the current limits for the safe use of ultrasound in cardiac applications $(\mathrm{MI}=2.26$, $\mathrm{I}_{\text {sppa }}=576 \mathrm{~W} \cdot \mathrm{cm}^{-2}$ and $\mathrm{I}_{\text {spta }}=1407 \mathrm{~mW} \cdot \mathrm{cm}^{-2}$ ), whereas a TI of 3.0 is considered safe for scanning times up to 4 minutes. Nonetheless, any ultrasonic sequence has to comply with the safety standards in order to be implemented clinically. Therefore, the increase in acoustic output of MLT beam forming

\footnotetext{
${ }^{1}$ In fact, for FDA approval, either MI or $\mathrm{I}_{\text {sppa }}$ parameters may exceed the regulatory limit.
} 

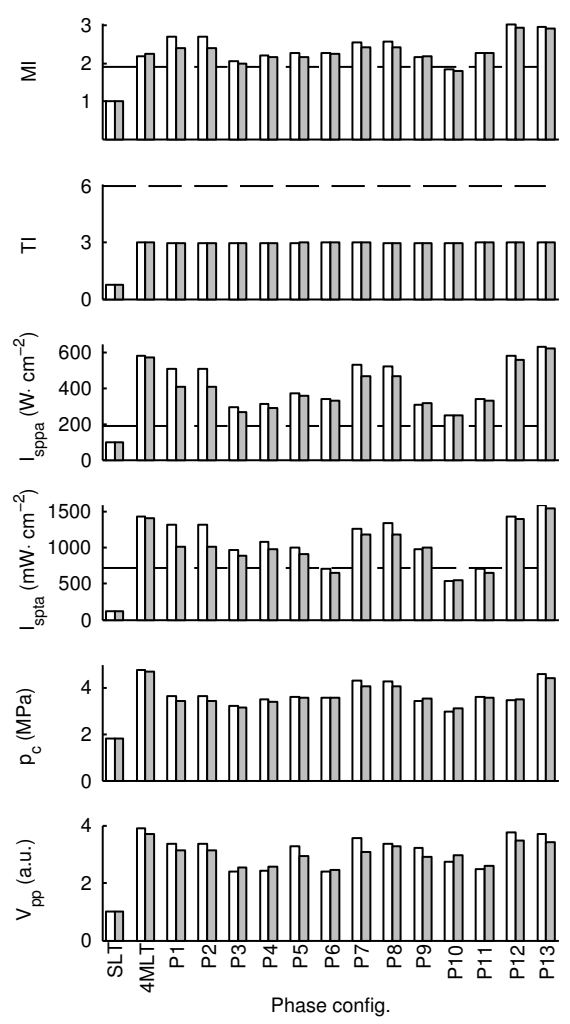
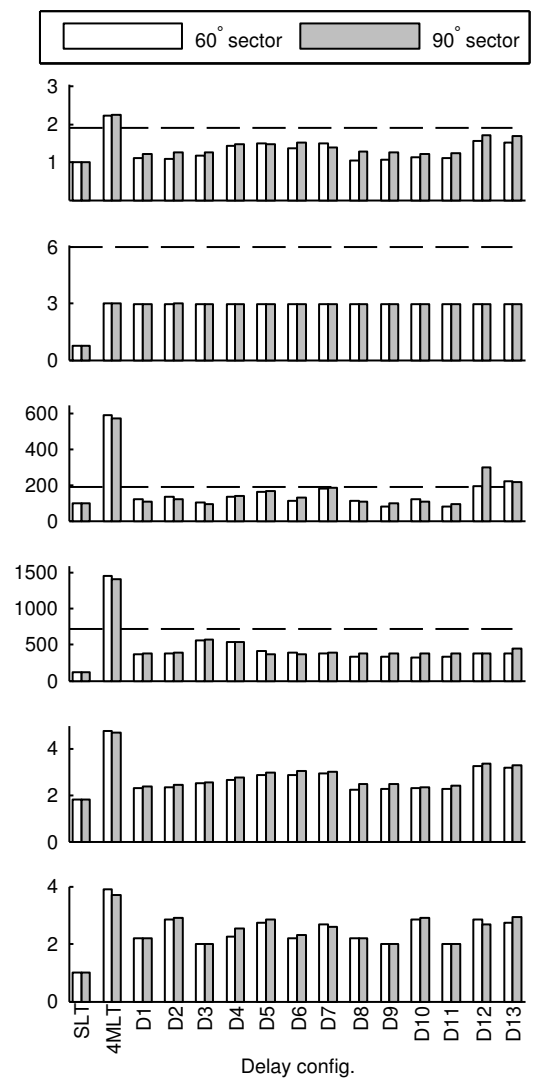
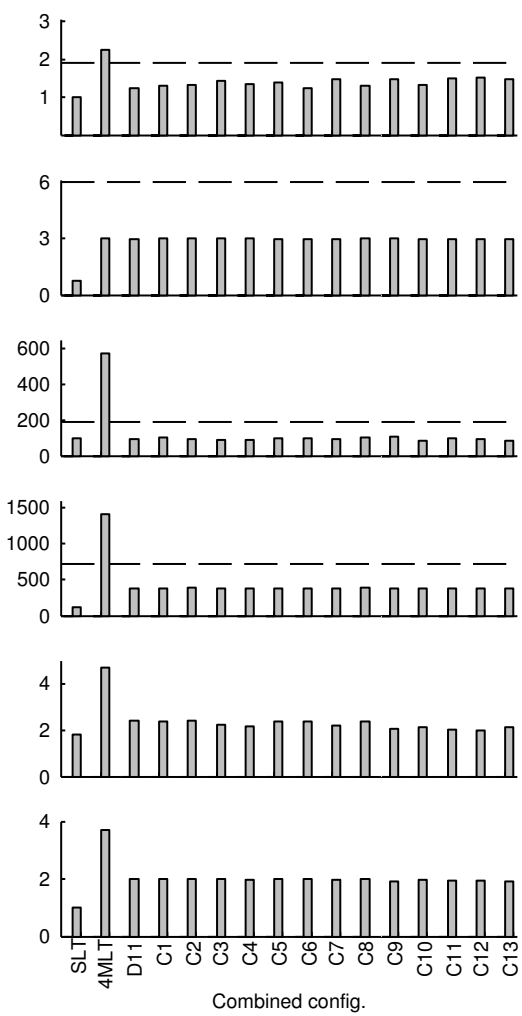

Fig. 4. Comparison of the safety parameters for phased 4-MLT (left column), delayed 4-MLT (middle column) and their combination (right column). MI, TI, $\mathrm{I}_{\text {sppa }}, \mathrm{I}_{\mathrm{spta}}, \mathrm{p}_{\mathrm{c}}$ and $\mathrm{V}_{\mathrm{pp}}$ are shown and their safety limit is shown by the dashed horizontal lines.

means that compromises have to be done on the transmit side: e.g. decrease transmit voltage, shorten the transmit pulses or lower the pulse repetition frequency (PRF). These concessions will assure an acoustic output below the safe levels at the expense of SNR and/or frame rate.

An additional concern in medical ultrasound is surface heating. An extensive study [35] has been presented on soft tissue temperature rise during focused scan modes (e.g. Bmode) and reported that the maximum temperature occurred at the skin surface for nearly all setups tested. These findings formed the basis of the definition of TIS [36]. Accordingly, the increase in TI shown for MLT beam forming anticipates an increase in tissue temperature at the surface. Nevertheless, TI values were compliant with regulatory levels.

Transducer surface heating depends on a vast number of pulse parameters (e.g. drive voltage, centre frequency, waveform) and scan sequence layout, as well as transducer construction details. Therefore, its prediction is very complex and strongly relies on experimental data [37]. As such, it was not considered in the present study and may be a topic for future work. Nonetheless, MLT is expected to result in higher surface temperature due to the transmission of multiple pulses in the same transmit event, although the exact temperature elevation is unknown at the moment. However, in case a practical implementation would exceed the regulatory levels, surface temperature could be decreased at the expense of SNR, by decreasing the $\mathrm{V}_{\mathrm{pp}}$.
All these particularities could limit the applicability of the technique. Hence, it is important to design the MLT sequence in a way that acoustic output is decreased without compromising (significantly) image quality. The modifications studied here might present a solution to mitigate these concerns.

\section{Phased MLT}

Pulse phase modifications led to changes in the interference pattern. Namely, beam interference could be changed in a way such that constructive interference for a given pair of beams was overlapping with destructive interference of another pair of beams in several configurations. Accordingly the configuration with the best results was the one with pulse phases of $\left[\frac{\pi}{2}-\pi-0-\frac{3 \pi}{2}\right]$. For this configuration, the two central beams are in opposite phase, and so are the outer two. Therefore, the interference that would in standard 4-MLT occur along the medial direction was cancelled for each pair of beams. Moreover, adjacent beams are transmitted with a $\frac{\pi}{2}$ phase shift, thereby further reducing acoustic interference.

Additionally, some of the configurations resulted in a slight shift of the peak pressure either to deeper or to more lateral positions (e.g. modification $\mathrm{P} 3$ and $\mathrm{P} 4$, where it was moved to around $5 \mathrm{~mm}$ deeper). As a result, the peak pressure moved along an arc during the scanning, as shown by the complete sequence (Media-Movie 1). This led to a slight spatial smoothing of intensity over the sector scan, thereby decreasing $\mathrm{I}_{\text {spta }}$. 

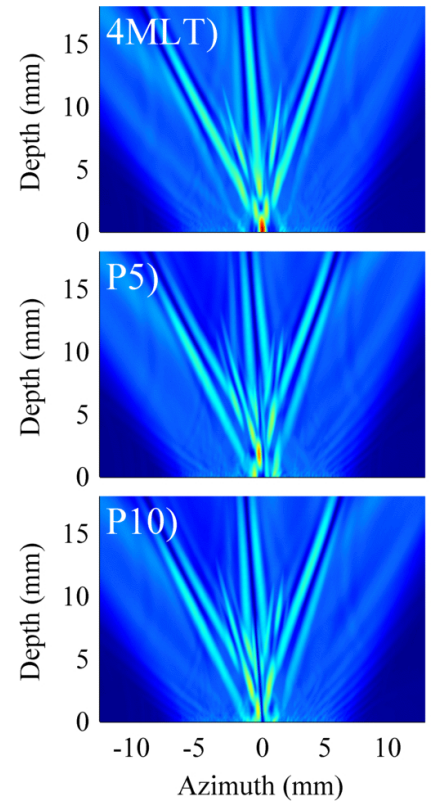
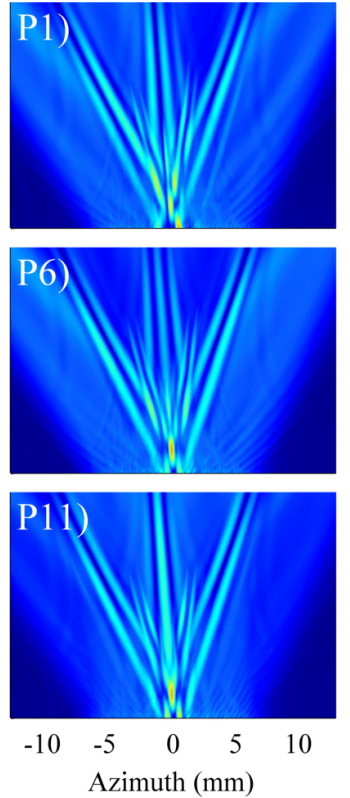
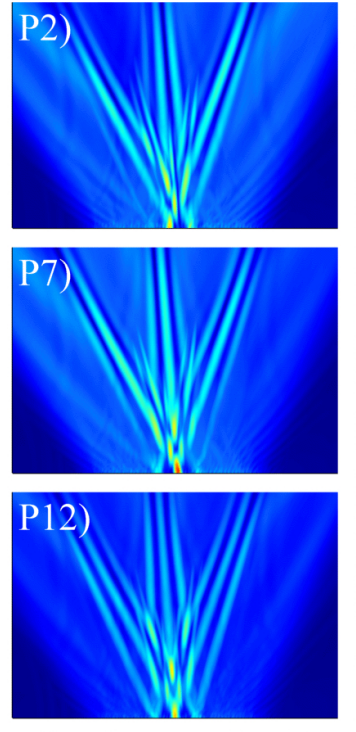

$\begin{array}{lllll}-10 & -5 & 0 & 5 & 10\end{array}$

Azimuth (mm)
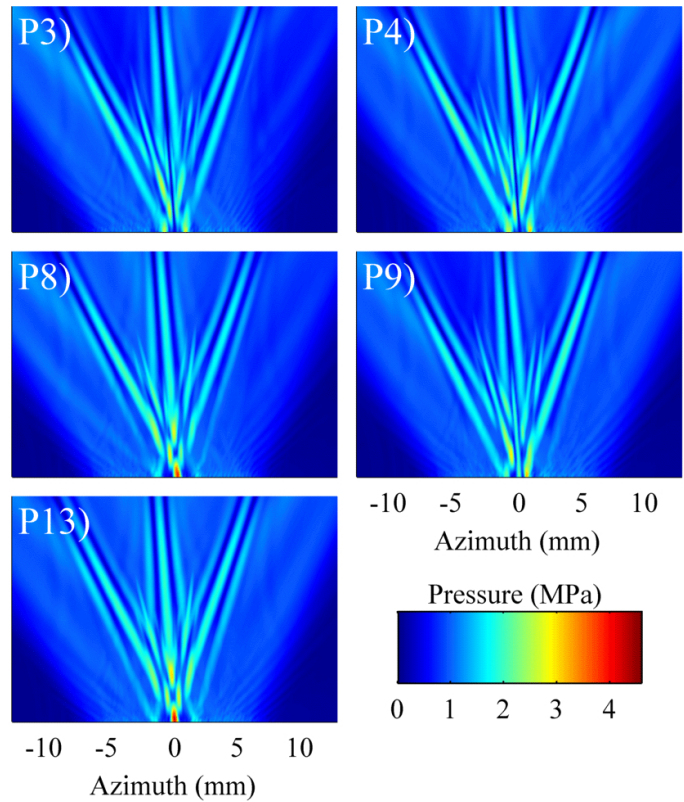

$\begin{array}{lllll}-10 & -5 & 0 & 5 & 10\end{array}$

Azimuth (mm)

Pressure (MPa)

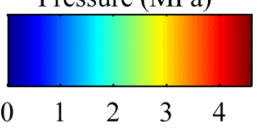

Fig. 5. Acoustic pressure for the pulse phase configurations described in Table III. Note that only the near field is plotted. Media-Movie 1

Indeed, P3 was next in terms of reduced acoustic parameters, with output values very close to P10. Interestingly, this configuration also has the two central beams with symmetric pulse phase. Moreover, as Fig. 4 shows, the best configurations tend to be the same for either sector angle.

All configurations showed reduced acoustic pressure, as well as acoustic intensity, in the region of beam overlap. For the best configuration, $\mathrm{p}_{\mathrm{c}}$ decreased by $33 \%$ while MI was reduced by $21 \%$, when compared to the standard implementation of 4-MLT. Although this modification does not change the pulse duration, the decrease in acoustic pressure was reflected in a decrease of $I_{\text {sppa }}$ by a factor of 2.3. Moreover, $I_{\text {spta }}$ decreased by a factor of 2.6 .

Several modifications resulted in an increased MI. This is not surprising, considering that the standard configuration (i.e. with all pulses with phase $\varphi_{0}=0$ ) has the highest positive amplitude, but not the highest negative one (Fig. 1). Hence, the highest MI occurred for the configuration P12, i.e. the one with all the multiple pulses having phases of $\varphi_{0}=\pi$. As mentioned in Section II-B3, the delays of the central element are the same for every MLT beam. Therefore, the global acoustic beam of P12 consists of multiple beams perfectly summing in front of the central element, each one of them emitted with the highest negative peak amplitude.

On the contrary, the TI remained the same as the one of standard 4-MLT. This was expected given that the pulse phase modification does not introduce major changes in the pulse shape nor magnitude. Therefore, the physical quantity behind TI - the total output power - remains fairly constant. Major changes in TI would only be expected with, e.g. decrease in PRF or transmit voltage.

$\mathrm{V}_{\mathrm{pp}}$ was decreased for all modifications, with the lowest value achieved for P6, being 2.47 higher than the one of SLT beam forming. Finally, phased MLT changes the effective pulse waveform (e.g. $\mathrm{V}_{\mathrm{pp}}$ and pulse duration), thus possibly influencing transducer surface heating.

Although the safety parameters of 4-MLT were reduced by this modification, only configuration P10 reached values of acoustic safety.

\section{Delayed MLT}

Reorganising the delay lines resulted in a considerable change in the global beam profile of 4-MLT. Namely, the overlap between certain pairs of beams was avoided in several configurations. Moreover, a pronounced shift of the lines of interference between multiple MLT beams was observed, as seen in Fig. 6. The shift of the interference axis during the sector scan (c.f. Media-Movie 2) results in a spatial smoothing of the average intensity. Additionally, the intersection of all delay lines in the central element was avoided, occurring now pairwise in more than one element, thereby spreading the acoustic output once more.

Delayed MLT showed a decrease of the acoustic output parameters considerably greater than the one achieved with the phased MLT. This result was expected, given that shifting the delay lines of the transmit event means a temporal - and consequently spatial - separation of the multiple transmitted beams. In fact, all the tested configurations were found to be safe (Fig. 4). Although configurations D12 and D13 did exceed the safety limit for $\mathrm{I}_{\text {sppa }}$, they did comply with the limit for MI. Altogether, the changes in the global acoustic field resulted in a reduction of the acoustic pressure parameters (MI and $\mathrm{p}_{\mathrm{c}}$ ) by at least $25 \%$ and intensity parameters $\left(\mathrm{I}_{\text {sppa }}\right.$ and $\left.\mathrm{I}_{\text {spta }}\right)$ by more than $60 \%$ (with the exception of D12). Decrease in $\mathrm{I}_{\text {sppa }}$ was also accomplished by the increase in pulse duration due to delaying some of the beams, according to (5). Alike the previous modification, no substantial decrease of TI was observed due to its dependence on the total acoustic output power and the tested configurations showed similar behaviour for both scan geometries. 

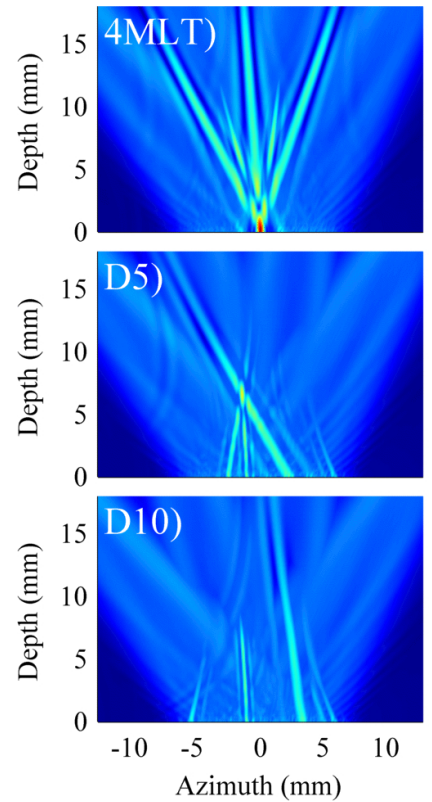
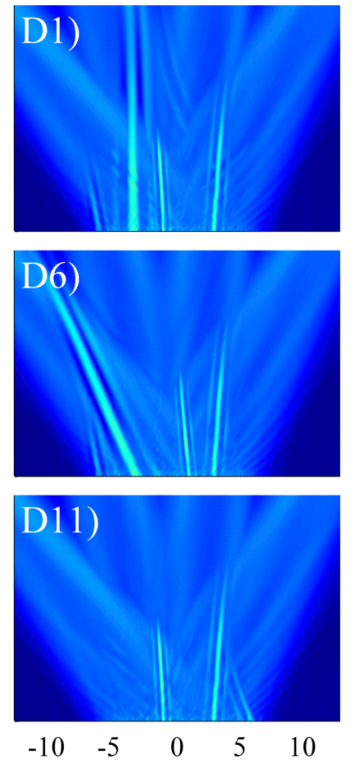

Azimuth (mm)
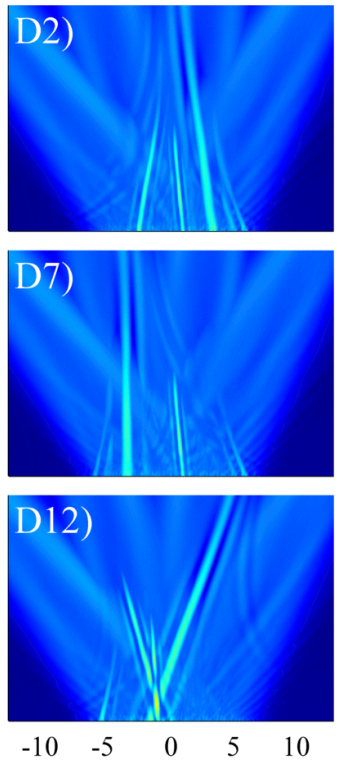

Azimuth (mm)
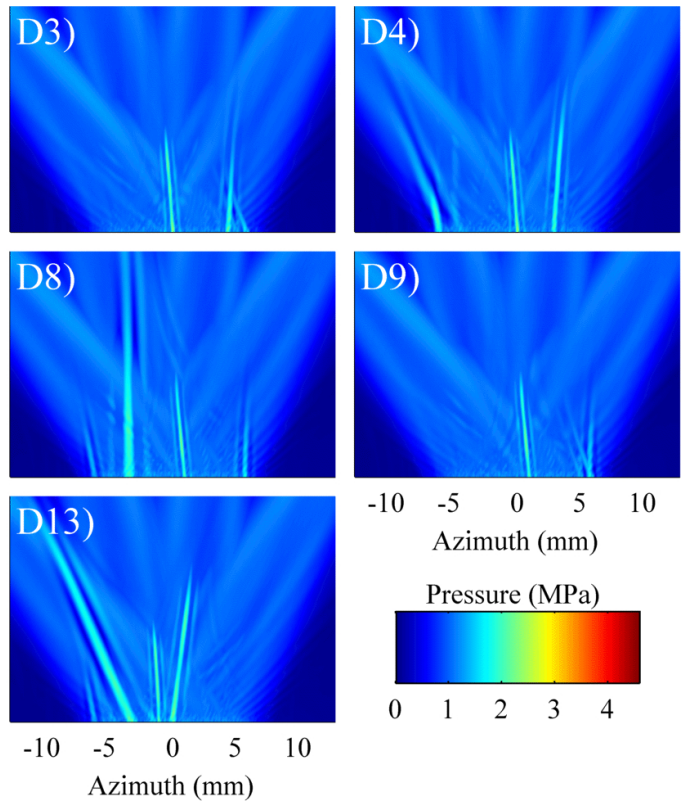

$\begin{array}{lllll}-10 & -5 & 0 & 5 & 10\end{array}$

Azimuth (mm)

Pressure (MPa)

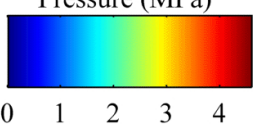

Fig. 6. Acoustic pressure for the pulse delay line configurations described in Table IV. Note that only the near field is plotted. Media-Movie 2

Additional decrease of the acoustic interference could be achieved by further separating the multiple beams at the expense of near field loss. In the limit, a configuration with all beams fully separated would require the last beam to be transmitted up to $17 \mu$ s after the beginning of the transmit event. This would imply a loss in near field of up to $2.6 \mathrm{~cm}$.

As delayed-MLT introduces considerable changes in the transmit pulse (e.g. fully separating pulses in some elements), probe heating signature may be modified.

Another interesting advantage of this is related to hardware restrictions and beam distortions. All systems have a limit on the maximum voltage that can be applied to each element of the transducer. Therefore, there is a compromise between the transmit voltage for elements where pulse summation occurs and for elements where it does not. With the standard 4-MLT beam forming approach used in this study, $\mathrm{V}_{\mathrm{pp}}$ at the central element is four times higher than the one on the remaining elements (Fig 2 - left panel). Hence, an excitation with the maximum voltage applied to this element implies a reduction on voltage of the other elements to $1 / 4$, thereby leading to a decrease in the transmitted energy and consequently in the SNR. Alternatively, an excitation with the full voltage on the remaining elements would imply clipping the voltage transmitted to the central pulse, thus resulting in possible distortions of the transmitted beam profile. Both approaches assume that a multi-level pulser is present on the echocardiographic system. When such pulser is not present (as typically happens in current clinical systems) all elements must be excited with the same $\mathrm{V}_{\mathrm{pp}}$, again leading to beam distortions. With the proposed modification, $V_{p p}$ was significantly reduced (Fig. 4), thus meaning an immediate improvement on the limitations described. Quantitatively, the configuration D3 was the one with the lowest $\mathrm{V}_{\mathrm{pp}}$ required - two times higher than SLT beam forming and one half of the one of standard 4-MLT.

\section{E. Combination of Both Modifications}

Results showed similar acoustic output values for all the pulse phases tested with the delayed configuration D11 (Fig. 4). This was especially evident for $I_{\text {spta }}$, for which the variation across the 14 configurations was only $3 \%$. The reason for much smaller variations is that the beams were already partially separated in space. Therefore, local beam phase changes have a much smaller influence.

Indeed, due to the fact that delay lines reorganisation also disrupts the spatial symmetry between beams, the change in the acoustic output parameters did not follow the same pattern as previously for pulse phase changes with no delays between MLT beams (c.f. Fig. 4 - first and last columns). As such, the configurations that led to a perfect destructive interference in the original modification, do not perform with the same outcome after applying delays to some of the beams.

Hence, practical implementation of these modifications may be done in a way that either (or both) the pulse phase per beam and the delay between MLT beams are allowed to adapt for each transmit event during the scan sequence. Optimization may be used to define the delay line alignment that results in less overlap between MLT beams, as well as the set of pulse phases that provides an efficient destructive interference, even when the beams are slightly delayed with respect to each other.

\section{F. Additional Remarks}

No configuration minimized all acoustic output parameters. Therefore, the choice of the specific configuration will depend on the desired acquisition scheme and imaging mode. In fact, different imaging modes might have different acoustic limitations. For instance, pulsed wave (PW) Doppler is more demanding in terms of $I_{\text {spta }}$ (due to the acquisition of a single direction) than B-mode imaging. Moreover, some safety parameters are measured differently for non-scanning modes 

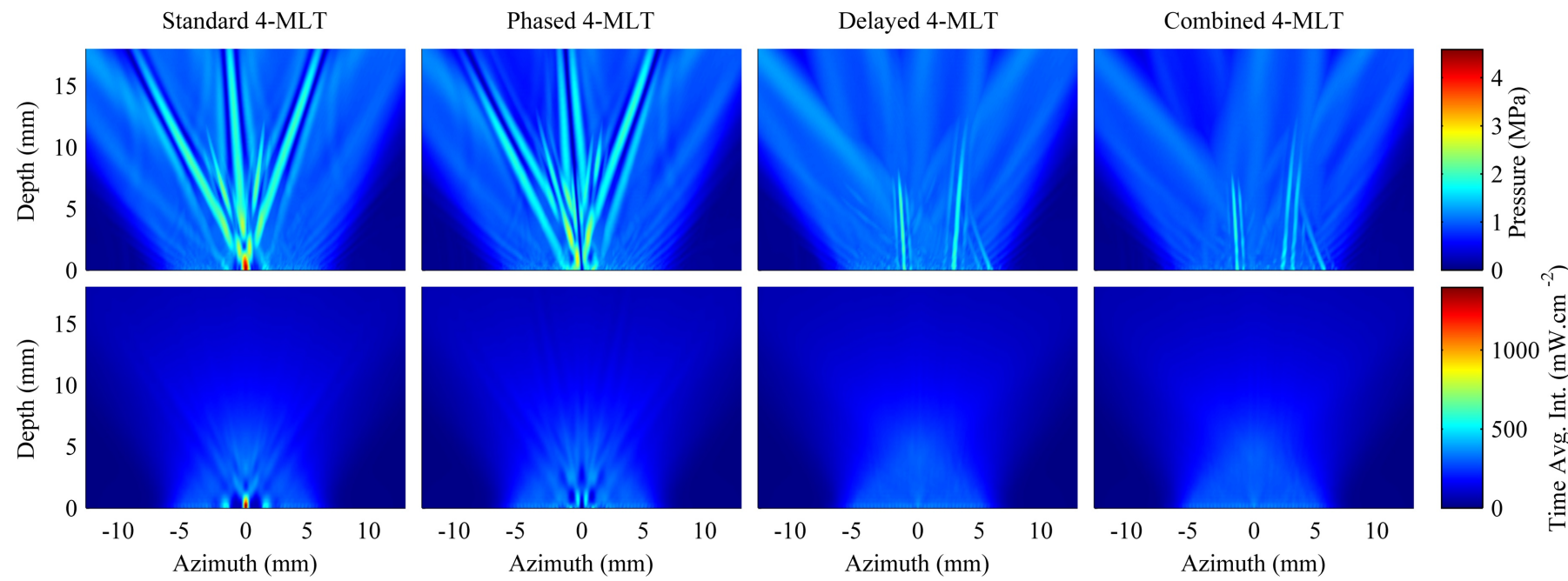

Fig. 7. Acoustic pressure of a given transmit event (top) and time averaged intensity for a sector scan (bottom) for four 4-MLT configurations. From left to right: standard 4-MLT, best phased configuration (P10), best delayed configuration (D11) and best combination of both modifications (C10). Note that only the near field is plotted. Media-Movie 3

(e.g. TI [25] and $\left.\mathrm{I}_{\text {spta }}[26]\right)$. The values presented in this study were computed for scanning modes (e.g. B-mode) and results show that MLT beam forming could potentially enable the temporal resolution required for many different cardiac studies - e.g. motion, deformation and elasticity imaging, mechanical activation imaging and contrast imaging [2], [3] - without significantly sacrificing SNR.

The reduction of the acoustic output in the near field of MLT beam forming is not limited to the modifications proposed. One alternative strategy could be the use of different frequencies for each pulse, as this would avoid perfect acoustic summation of all MLT beams. This idea has already been proposed with the aim of improving image quality by reducing the cross-talk artifacts [38]. Nonetheless, frequencies of the MLT beams should preferably be kept relatively similar, in order to avoid different frequency-dependent attenuation. Moreover, different focal depths could be given to each MLT beam. This would allow for a change in the curvature of the individual delay lines, thus changing the interference pattern. Again, for the sake of image quality, focal depths should be fairly similar, as this influences spatial resolution. Obviously, $\mathrm{TI}$ and $\mathrm{I}_{\text {spta }}$ could alternatively be reduced by decreasing the PRF. This would however decrease the effective frame rate.

Finally, this study was focused exclusively on the acoustic parameters computed from the transmit beam profile. Further studies should be conducted on the two-way beam profile, namely to assess whether these modifications lead to any significant decrease in imaging quality. A final solution would most probably be a compromise between frame rate, imaging quality and acoustic safety, being the last one absolutely imperative.

\section{CONClusion}

4-MLT beam forming was found to substantially elevate the acoustic output when compared to SLT. Accordingly, it exceeds the current safety thresholds if implemented in the standard way (i.e. linearly summing 4 SLT transmit events).
Therefore, compromises would have to be made on the transmit sequence (e.g. lower the transmit voltage) in order for it to comply with safety standards and be implemented clinically. However, such concessions might reduce significantly the SNR and tissue penetration, thereby limiting its applicability. This is of greater concern for Doppler modes, which typically have higher TI and $\mathrm{I}_{\text {spta }}$ than B-mode. Hence, two modifications of the standard 4-MLT were implemented: i) generating out of phase pulses for each MLT beam and ii) reorganizing the delay lines of the transmit event. These modifications resulted in a more uniform distributing of the acoustic energy, thus allowing for a decrease of the safety parameters related to spatial peaks to levels in the order of those of SLT beam forming. Drive voltage amplitude required was also considerably reduced. On the contrary, no major changes were achieved for TI, although its values were compliant with the safety guidelines for short scanning times. Therefore, this work helps mitigating the concerns of MLT beam forming, significantly reducing the potential trade-offs required for it to be safely used in clinical imaging.

\section{ACKNOWLEDGMENTS}

The research leading to these results is supported by the European Union under a Marie Curie Initial Training Network (USART-project, Grant Agreement PITN-GA-2012317132) and by the European Research Council under the European Unions Seventh Framework Programme (FP7/20072013, ERC Grant Agreement 281748).

\section{REFERENCES}

[1] H. Kanai and Y. Koiwa, "Myocardial rapid velocity distribution," Ultrasound in medicine \& biology, vol. 27, no. 4, pp. 481-498, Apr. 2001.

[2] M. Cikes, L. Tong, G. R. Sutherland, and J. D'hooge, "Ultrafast Cardiac Ultrasound Imaging: Technical Principles, Applications, and Clinical Benefits." Journal of the American College of Cardiology: Cardiovascular Imaging, vol. 7, no. 8, pp. 812-823, Aug. 2014.

[3] M. Tanter and M. Fink, "Ultrafast imaging in biomedical ultrasound," Ultrasonics, Ferroelectrics, and Frequency Control, IEEE Transactions on, vol. 61, no. 1, pp. 102-119, Jan. 2014. 
[4] R. R. Bouchard, S. J. Hsu, M. L. Palmeri, N. C. Rouze, K. R. Nightingale, and G. E. Trahey, "Acoustic radiation force-driven assessment of myocardial elasticity using the displacement ratio rate (DRR) method." Ultrasound in medicine \& biology, vol. 37, no. 7, pp. 1087-100, Jul. 2011.

[5] B. Brekke, L. C. L. Nilsen, J. Lund, H. Torp, T. Bjåstad, B. H. Amundsen, A. Stoylen, and S. A. Åse, "Ultra-high frame rate tissue Doppler imaging," Ultrasound in medicine \& biology, vol. 40, no. 1, pp. 222-31, Jan. 2014.

[6] S. Fadnes, S. A. Nyrnes, H. Torp, and L. Løvstakken, "Shunt Flow Evaluation in Congenital Heart Disease Based on Two-Dimensional Speckle Tracking," Ultrasound in Medicine \& Biology, vol. 40, no. 10, pp. 2379-2391, Oct. 2014.

[7] M. Pernot and E. Konofagou, "Electromechanical imaging of the myocardium at normal and pathological states," in Ultrasonics Symposium (IUS), 2005 IEEE International, vol. 2, 2005, pp. 1091-1094.

[8] D. P. Shattuck, M. D. Weinshenker, S. W. Smith, and O. T. von Ramm, "Explososcan: a parallel processing technique for high speed ultrasound imaging with linear phased arrays." The Journal of the Acoustical Society of America, vol. 75, no. 4, pp. 1273-82, Apr. 1984.

[9] J. D'hooge, E. E. Konofagou, F. Jamal, A. Heimdal, L. Barrios, B. Bijnens, J. Thoen, F. Van de Werf, G. R. Sutherland, and P. Suetens, "Two-dimensional ultrasonic strain rate measurement of the human heart in vivo," Ultrasonics, Ferroelectrics and Frequency Control, IEEE Transactions on, vol. 49, no. 2, pp. 281-286, Feb. 2002.

[10] M. Pernot, K. Fujikura, S. D. Fung-Kee-Fung, and E. E. Konofagou, "ECG-gated, mechanical and electromechanical wave imaging of cardiovascular tissues in vivo." Ultrasound in medicine \& biology, vol. 33, no. 7, pp. 1075-85, Jul. 2007.

[11] L. Sandrin, M. Tanter, S. Catheline, and M. Fink, "Shear modulus imaging with 2-D transient elastography," Ultrasonics, Ferroelectrics and Frequency Control, IEEE Transactions on, vol. 49, no. 4, pp. 426435, Apr. 2002.

[12] H. Hasegawa and H. Kanai, "High-frame-rate echocardiography using diverging transmit beams and parallel receive beamforming," Journal of Medical Ultrasonics, vol. 38, no. 3, pp. 129-140, Jul. 2011.

[13] G. Montaldo, M. Tanter, J. Bercoff, N. Benech, and M. Fink, "Coherent plane-wave compounding for very high frame rate ultrasonography and transient elastography," Ultrasonics, Ferroelectrics and Frequency Control, IEEE Transactions on, vol. 56, no. 3, pp. 489-506, Apr. 2009.

[14] C. Papadacci, M. Pernot, M. Couade, M. Fink, and M. Tanter, "Highcontrast ultrafast imaging of the heart," Ultrasonics, Ferroelectrics and Frequency Control, IEEE Transactions on, vol. 61, no. 2, pp. 288-301, Feb. 2014

[15] T. Shirasaka, "Ultrasonic imaging apparatus," US Patent 4,815,043, Mar. 1989.

[16] F. Prieur, B. Dénarié, A. Austeng, and H. Torp, "Multi-line transmission in medical imaging using the second-harmonic signal," Ultrasonics, Ferroelectrics, and Frequency Control, IEEE Transactions on, vol. 60, no. 12, pp. 2682-2692, Dec. 2013.

[17] R. A. Snyder, "Ultrasound imaging system utilizing two or more simultaneously-active apertures," European Patent EP0335578 A3, Aug. 1990.

[18] R. Mallart and M. Fink, "Improved imaging rate through simultaneous transmission of several ultrasound beams," New Developments in Ultrasonic Transducers and Transducer Systems, vol. 1733, pp. 120-130, Nov. 1992.

[19] B. Madore, P. J. White, K. Thomenius, and G. T. Clement, "Accelerated focused ultrasound imaging," Ultrasonics, Ferroelectrics and Frequency Control, IEEE Transactions on, vol. 56, no. 12, pp. 2612-23, Dec. 2009.

[20] L. Tong, A. Ramalli, R. Jasaityte, P. Tortoli, and J. D'Hooge, "MultiTransmit Beam Forming for Fast Cardiac Imaging - Experimental Demonstration and In-Vivo Application," Medical Imaging, IEEE Transactions on, vol. 33, no. 6, pp. 1205-1219, Jun. 2014.

[21] B. Dénarié, T. Bjåstad, and H. Torp, "Multi-Line Transmission in 3-D With Reduced Crosstalk Artifacts: A Proof of Concept Study," Ultrasonics, Ferroelectrics and Frequency Control, IEEE Transactions on, vol. 60, no. 8, pp. 1708-1718, Aug. 2013.

[22] L. Demi, J. Viti, and L. Kusters, "Implementation of parallel transmit beamforming using orthogonal frequency division multiplexingachievable resolution and interbeam interference," Ultrasonics, Ferroelectrics and Frequency Control, IEEE Transactions on, vol. 60, no. 11, pp. 2310-2320, Nov. 2013.

[23] AIUM/NEMA, Standard for Real-Time Display of Thermal and Mechanical Acoustic Output Indices on Diagnostic Ultrasound Equipment. American Institute of Ultrasound in Medicine, and National Electrical Manufacturers Association, 2009.
[24] AIUM, "Bioeffects Considerations for the Safety of Diagnostic Ultrasound," Journal of Ultrasound in Medicine, vol. 7, no. 9, Supp., pp. S13-38, 1988

[25] IEC, IEC 60601-2-37 - Particular requirements for the safety of ultrasonic medical diagnostic and monitoring equipment, 1st ed. Geneva: International Electrotechnical Commission, 2004.

[26] FDA, Information for manufacturers seeking marketing clearance of diagnostic ultrasound systems and transducers. US Food and Drug Administration, 2008, vol. 62, no. 114.

[27] K. E. Thiele, "Multi Beam Transmit Isolation,” US Patent 2010/0016725, 2010.

[28] P. Santos, L. Tong, A. Ortega, L. Løvstakken, E. Samset, and J. D’hooge, "Safety of Multi-Line Transmit beam forming for fast cardiac imaging - a simulation study," Ultrasonics Symposium (IUS), 2014 IEEE International, pp. 1199-1202, 2014.

[29] J. D'hooge, J. Nuyts, B. Bijnens, B. De Man, P. Suetens, J. Thoen, M.-C. Herregods, and F. Van de Werf, "The calculation of the transient near and far field of a baffled piston using low sampling frequencies," The Journal of the Acoustical Society of America, vol. 102, no. 1, pp. 78-86, Jul. 1997.

[30] L. Tong, H. Gao, and J. D'hooge, "Multi-transmit beam forming for fast cardiac imaging-a simulation study," Ultrasonics, Ferroelectrics and Frequency Control, IEEE Transactions on, vol. 60, no. 8, pp. 1719-1731, Aug. 2013.

[31] IEC, IEC 62359 - Ultrasonics - Field characterization - Test methods for the determination of thermal and mechanical indices related to medical diagnostic ultrasonic fields, 2nd ed. Geneva: International Electrotechnical Commission, 2010.

[32] - IEC 62127-1 - Ultrasonics - Hydrophones - Part 1: Measurement and characterization of medical ultrasonic fields up to $40 \mathrm{MHz}$, 1st ed. Geneva: International Electrotechnical Commission, 2013.

[33] K. Beissner, "On the Plane-Wave Approximation of Acoustic Intensity," Journal of the Acoustical Society of America, vol. 71, no. 6, pp. 14061411, Jun. 1982.

[34] BMUS, Guidelines for the safe use of diagnostic ultrasound equipment. British Medical Ultrasound Society, 2009.

[35] M. G. Curley, "Soft tissue temperature rise caused by scanned, diagnostic ultrasound," Ultrasonics, Ferroelectrics and Frequency Control, IEEE Transactions on, vol. 40, no. 1, pp. 59-66, Jan. 1993.

[36] J. G. Abbott, "Rationale and derivation of MI and TI-a review," Ultrasound Med Biol, vol. 25, no. 3, pp. 431-441, Mar. 1999.

[37] W. S. Ohm, J. H. Kim, and E. C. Kim, "Prediction of surface temperature rise of ultrasonic diagnostic array transducers," Ultrasonics, Ferroelectrics and Frequency Control, IEEE Transactions on, vol. 55, no. 1, pp. 125-138, Jan. 2008.

[38] L. Demi, M. D. Verweij, and K. W. Van Dongen, "Parallel transmit beamforming using orthogonal frequency division multiplexing applied to harmonic imaging-a feasibility study," Ultrasonics, Ferroelectrics and Frequency Control, IEEE Transactions on, vol. 59, no. 11, pp. 24392447, Nov. 2012.

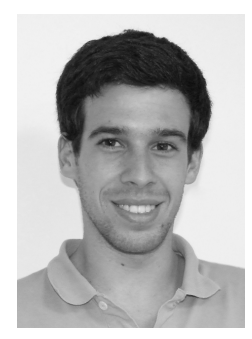

Pedro Santos was born in Penacova, Portugal, in 1989. He received the M.Sc. degree in Biomedical Engineering from the University of Coimbra, Portugal, in September 2012. He integrated the Electronics and Instrumentation Group of that university in 2009, where he was committed to research in Hemodynamics. In 2013, he joined the Ultrasound-guided Cardiac Arrhythmia Treatment project (USART http://www.usart-project.eu) as a Ph.D. student (KU Leuven). His topic is fast cardiac imaging, focusing on electromechanical wave detection. 


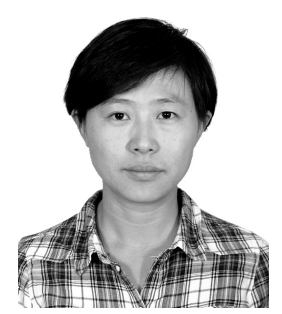

Ling Tong was born in Hebei, China, in 1983. She received her Bachelor degree in Physics from Hebei University, China in 2006, her Master degree in Condensed Matter Physics from Peking University, China in 2009, and her Ph.D. degree from the Department of Cardiovascular Sciences in the University of Leuven (KU Leuven), Belgium in 2013. Since 2014, she started working as a postdoc researcher in the Department of Biomedical Engineering in Tsinghua University, China. Her main research field is ultrasound beamforming and signal processing for high frame rate cardiac imaging.

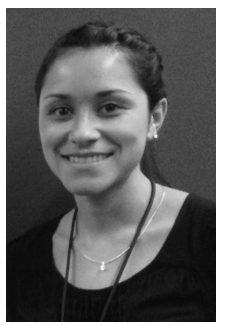

Alejandra Ortega was born in Medellin, Colombia the $16^{\text {th }}$ of May of 1988. She studied biomedical engineering at the Antioquia School of Engineering University. During her bachelor she was part of Biomedical Engineering Research Group EIACES (GIBEC), with emphasis in medical imaging. Also, Alejandra did six months of internship in the Magnetic Resonance Elastography Laboratory, at Mayo Clinic (Rochester, MN); there, she was part of different projects, including acquisition and processing of the MRI images. Her undergraduate thesis was entitled "Compiled and compared monograph between MRI and US in the estimation of tissues elasticity". Currently, Alejandra is doing her doctoral studies on fast cardiac volumetric imaging at KU Leuven.

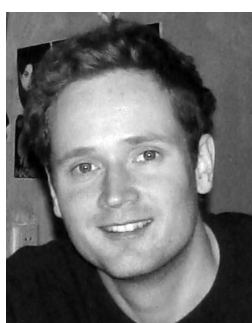

Lasse Løvstakken was born in Bergen, Norway, in 1976. He received his M.Sc. degree in engineering cybernetics and his Ph.D. degree in medical technology from the Norwegian University of Science and Technology in Trondheim in 2002 and 2007, respectively. He is currently Professor at the Department of Circulation and Medical Imaging, Norwegian University of Science and Technology. His research interests include image formation and signal processing, with emphasis on blood flow imaging in diagnostic ultrasound.

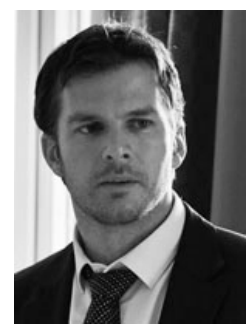

Eigil Samset is a research project co-ordinator in GE Vingmed Ultrasound and Professor at the University of Oslo. He obtained his MSc from NTNU in Trondheim in 1997 in the field of engineering cybernetics. As a part of his studies he was a visiting researcher at Standford University (California, USA), in the Center for Research in Cardiovascular Interventions, studying intravascular ultrasound (IVUS). Dr. Samset received his $\mathrm{PhD}$ from the Faculty of Medicine at the University of Oslo in the field of MRI-guided therapy in 2003. He performed a 2 year post doctoral fellowship at Brigham and Women's Hospital in Boston (Massachusetts, USA). Currently, Eigil Samset is co-ordinating several research programs, including a centre for research driven innovation focusing on innovations in cardiac triage and treatment.

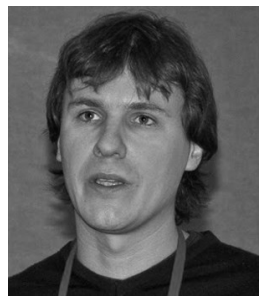

Jan D'hooge was born in Sint-Niklaas, Belgium, in 1972. He received the M.Sc. and Ph.D. degrees in physics at the University of Leuven (KU Leuven), Belgium, in 1994 and 1999, respectively. His dissertation studied the interaction of ultrasonic waves and biological tissues by means of computer simulation. He subsequently worked as a postdoctoral researcher at the Medical Imaging Computing Laboratory of the University of Leuven, where he became acquainted with general problems in medical imaging, such as elastic registration, segmentation, shape analysis, and data acquisition problems related to other modalities (in particular, MRI). During this period, he did part of the training for a bachelor's degree in biomedical sciences as a free student. In 2006, he was appointed an associate professorship in the department of cardiovascular diseases of the medical faculty. He is a member of the Acoustical Society of America, IEEE, and the European Association of Echocardiography. In 1999, he won the Young Investigator Award of the Belgian Society of Echocardiography, and in 2000, he was nominated for the Young Investigator Award of the European Society of Echocardiography. Since 2009, he has been a part-time visiting professor at the Norwegian Institute of Science and Technology (Trondheim, Norway). He was the chair of the ultrasound conference of the SPIE medical imaging symposium from 2008 until 2011, technical vice-chair of the IEEE Ultrasonics Symposium from 2008 to 2012 and the technical chair of the IEEE Ultrasonics Symposium 2014. He was an elected AdCom member of the IEEE-UFFC Society from 2010 to 2012 . He is (co)-author of more than 150 peer-reviewed papers, has contributed to 8 books, and has co-edited one book. His current research interests include myocardial tissue characterization, deformation imaging, and cardiac patho-physiology. 